\title{
Wavelet ridge diagnosis of time-varying elliptical signals with application to an oceanic eddy
}

\author{
J. M. Lilly ${ }^{1}$ and J.-C. Gascard ${ }^{2}$ \\ ${ }^{1}$ Earth and Space Research, Seattle, USA \\ ${ }^{2}$ Laboratoire d'Océanographie Dynamique et de Climatologie, Université Pierre et Marie Curie, Paris, France
}

Received: 8 May 2006 - Revised: 29 August 2006 - Accepted: 31 August 2006 - Published: 14 September 2006

\begin{abstract}
A method for diagnosing the physical properties of a time-varying ellipse is presented. This essentially involves extending the notion of instantaneous frequency to the bivariate case. New complications, and possibilities, arise from the fact that there are several meaningful forms in which a time-varying ellipse may be represented. A perturbation analysis valid for the near-circular case clarifies these issues. Diagnosis of the ellipse properties may then be performed using wavelet ridge analysis, and slowly-varying changes in the ellipse structure may be decoupled from the fast orbital motion through the use of elliptic integrals, without the need for additional explicit filtering. The theory is presented in parallel with an application to a position time series of a drifting subsurface float trapped in an oceanic eddy.
\end{abstract}

\section{Introduction}

Many interesting time series are intrinsically bivariate. If the two variables represent the same physical quantity, such as a position or a velocity, or may be normalized in some meaningful way, then it is natural to think of the time series as tracing out an ellipse. Some examples of signals of this type include: planetary orbits, two-dimensional oscillators, measurements of currents or wind, electromagnetic polarization, and the three orthogonal planes of seismic motion. In the fields of oceanography and atmospheric science, ellipses are so common that the so-called rotary Fourier analysis (Gonella, 1972) is among the most widely used analysis tools. As with all Fourier analysis, however, the latter method is designed for stationary time series, and is therefore most appropriate for treating constant-amplitude, constantfrequency ellipses having random properties.

Correspondence to: J. M. Lilly

(lilly@esr.org)
Developments over the past decade or so have led to a wide range of new possibilities for treating non-stationary univariate or multivariate time series. In particular, diagnosis of the time-varying amplitude and frequency of a modulated oscillatory signal may be accomplished through wavelet ridge analysis (Delprat et al., 1992; Mallat, 1999), even if the signal is embedded in noise or other variability. This analysis is based on the notion of an asymptotic or weakly-modulated signal, for which the signal phase varies much more rapidly than the amplitude. In the absence of noise or other signal components, one may assign to such a signal a unique timevarying amplitude and phase pair directly through construction of the so-called analytic signal (Boashash, 1992). The wavelet ridge analysis essentially combines this construction with a series of bandpass operations, permitting it to succeed under a much broader range of conditions than the direct method.

The generalization of asymptotic signals, and their detection via the wavelet transform, to the case of a time-varying ellipse involves two important new issues. The first involves the fact that a time-varying ellipse may be decomposed in several different ways, with associated different conditions that the component time series be weakly modulated. This means that the information of greatest interest may be impossible to diagnose directly, but may still be inferred from another perspective. The second is that, after determining the ellipse parameters, important time-varying properties may be automatically averaged over one orbit of the ellipse through the use of elliptic integrals. This reflects the fact that an asymptotic signal, in which the phase must vary rapidly, captures the spirit of viewing a bivariate signal as an ellipse which is orbited rapidly relative to variations in its geometry.

As a sample application, we present data from a freedrifting subsurface oceanographic float trapped inside a coherent eddy structure. On the scale of tens of kilometers, the ocean abounds with energetic, organized vortices

Published by Copernicus GmbH on behalf of the European Geosciences Union and the American Geophysical Union. 
(McWilliams, 1985). These eddies may be deformed into ellipses by a number of phenomenon, most fundamentally, by the presence of an exterior strain field (Ruddick, 1987). This data presents an ideal application for this method. A time-varying elliptical signal is the dominant feature, with little other organized variability, yet the substantial frequency modulations preclude a Fourier-based analysis. Many hundreds of such data records exist, forming one of the most significant datasets for understanding the structure of the ocean on these scales. The creation of objective and informative analysis methods is therefore a subject of active research in the oceanographic community (e.g. Veneziani et al., 2005; Lankhorst and Zenk, 2006). Finally, the ellipse properties have exact and physically meaningful fluid dynamical interpretations.

Because of the broad potential applicability of this model, our approach will be general, aimed at clarifying the nature of the information which resides in a time-varying elliptical signal and best means for extracting it. Theoretical developments will be illustrated throughout with reference to the eddy-trapped float, rather than deferring the application section until the end. A perturbation analysis in powers of a variable related to the eccentricity is used to create simplified and highly accurate approximations which shed light on the interpretation of various quantities. The most significant exclusion is that we do not treat the role of noise or randomness in distorting results, but leave this important issue to a later work. Further, as the focus of present work is the fundamental method and not the practical problem of analyzing float trajectories, a comparison of this approach to studying Lagrangian data with the many other existing perspectives is also postponed.

Publication of this paper coincides with a public release of a complete software package, written in Matlab, for implementing all important equations, performing all analyses, and generating all figures. This software includes a number of innovative features designed for producing high-quality results from large datasets, and is released for use, modification, and redistribution by the community. Notes on the important aspects of this software package are included in Appendix C.

The structure of the paper is as follows. Section 2 introduces the data to be analyzed together with a preview of the analysis results. A brief review of the mathematical methods is presented in Sect. 3. Section 4 examines the representation and detection of time-varying elliptical signals using asymptotic signal components as a foundation. This raises some questions about the interrelationships between different representations, which are addressed in Sect. 5 through the use of a perturbation expansion valid for the near-circular regime. Section 6 concerns the construction of important instantaneous and average physical properties, including using the elliptic integrals to average over a period with fixed geometry. The paper concludes with a discussion.

\section{A motivational example}

In this section a data example is shown, which provides both a motivation for the development to follow, as well as a concrete reference point.

The data to be analyzed are shown in Fig. 1a. This time series is a horizontal position record from a Lagrangian, or freely drifting, oceanographic subsurface float of the type described by Rossby et al. (1986). This particular float tracked water motions in the eastern subtropical Atlantic during six months of 2001 as it drifted at 200-400 m depth. Its position was determined every four hours by triangulating reception times of signals from multiple fixed sound sources in the region.

Clearly visible in this record is a roughly circular signal traversed in a clockwise manner - superimposed on a lowerfrequency meander together with high-frequency instrument noise. Thus one would like a decomposition of the form

$z(t) \equiv x(t)+i y(t)=z_{e}(t)+z_{r}(t)$

$[i \equiv \sqrt{-1}]$ where $z_{e}(t)$ is a time-varying elliptical signal and $z_{r}(t)$ is a residual. The elliptical signal is believed to be associated with a so-called coherent eddy, an intense and longlived vortex thought to be an important element of the ocean circulation at scales on the order of ten kilometers. The degree of eccentricity has a number of possible interpretations, and in particular could reflect the magnitude of a background strain field (e.g. Ruddick, 1987); see our Appendix B.

The problem is to decouple the eddy signal from the other variability, and in this way to obtain estimates of the physical properties of the eddy itself. The ellipses obtained by our eventual decomposition, shown in Fig. 1b, reveal variations in ellipse amplitude, eccentricity, and orientation in far more detail than is possible to see from the original data plot. These variations could either mean changes in the eddy structure itself, or displacements of the float to different radial positions within the eddy.

The $x$ and $y$ position time series are shown in Fig. 2, together with our decomposition into an elliptical potion plus a residual. One sees large modulations of both the amplitude and the frequency of oscillatory original signal, as well as large fluctuations in the apparent local mean of this signal. This means that methods based around the assumption of a fixed frequency (e.g. Fourier analysis) or a nearly fixed frequency (e.g. complex demodulation) will fail to accurately separate the flucuations in the elliptical signal from those of its time-varying mean. By contrast, the wavelet ridge method we develop here yields a residual which appears to be almost completely devoid of the main oscillatory signal, even though the residual itself presents substantial variability.

The decomposition of Fig. 2 reflects the remarkable power of the wavelet ridge method. The challenge for a twocomponent time series is to understand how the information encoded in the ridges relates to the physical structure of a 

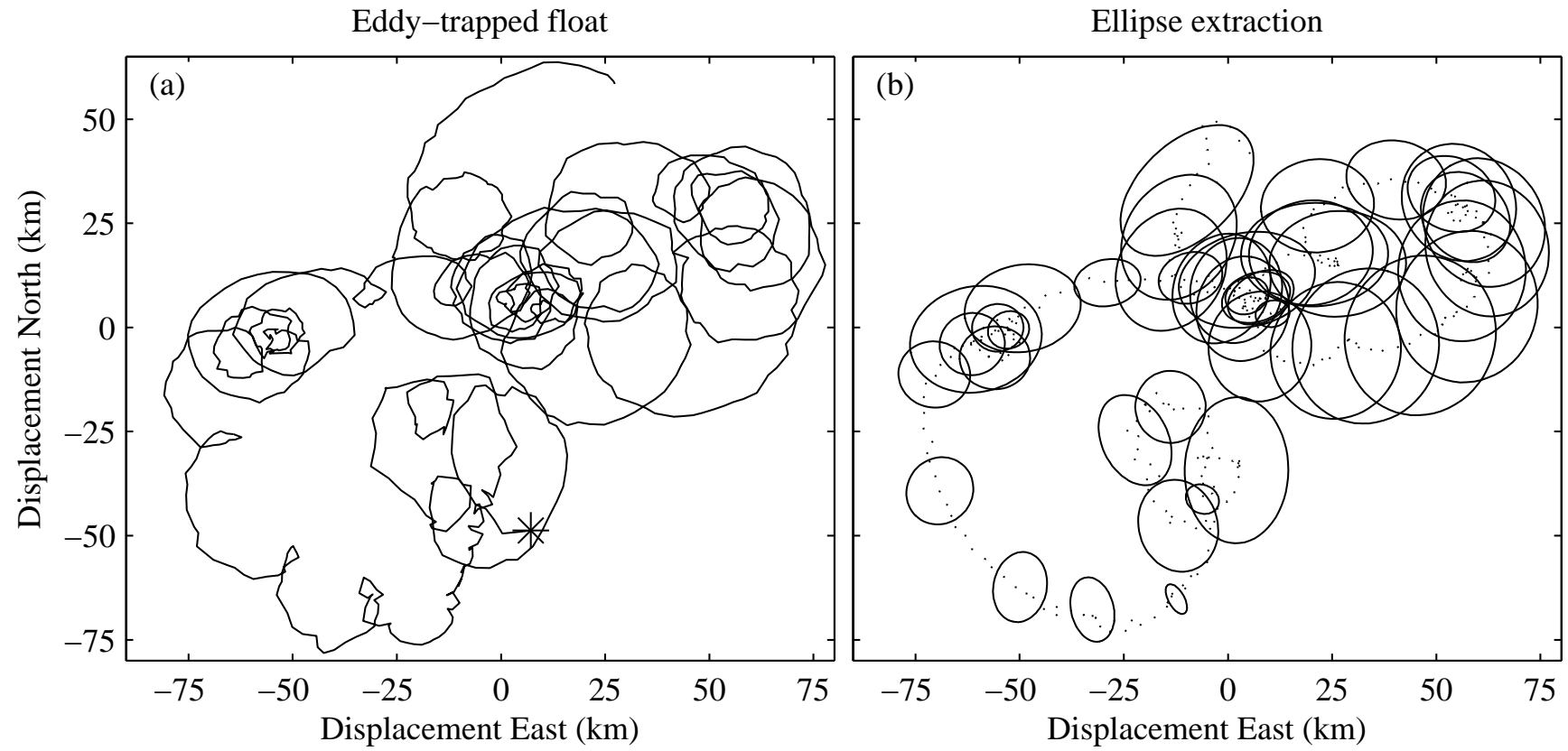

Fig. 1. A trajectory of a float trapped in an eddy (a), together with the decomposition of this signal into a time-varying elliptical signal plus a residual (b). In (a), the beginning of the time series is marked with an asterisk. In (b), the dotted line in the residual, and an ellipse is plotted every four days centered on the residual curve.

time-varying ellipse, and to identify the best way that information may be accessed. That is the purpose of this paper. At this point we set the data aside and turn to the development of the method.

\section{Background}

This section briefly reviews some important aspects of weakly-modulated signals and their detection using the continuous wavelet transform.

\subsection{The analytic signal}

A frequency- and amplitude-modulated real-valued signal may be written as

$x(t)=A(t) \cos (\phi(t))$

where the signal amplitude $A(t)$ is defined to be nonnegative. Because this is a powerful model, encompassing a broad range of interesting signals, one would like to unambiguously determine an amplitude and phase function from a signal $x(t)$. While the decomposition (Eq. 2) is not unique, a particular amplitude and phase may be uniquely assigned to $x(t)$ via an associated complex-valued signal

$x_{+}(t)=A_{x}(t) e^{i \phi_{x}(t)}$

called the analytic signal (Boashash, 1992).
The analytic signal may be uniquely constructed for a given $x(t)$ as described in Appendix A, with the original signal being recovered by

$x(t)=\Re\left\{x_{+}(t)\right\}=A_{x}(t) \cos \left(\phi_{x}(t)\right)$

where the amplitude $A_{x}(t)$ and phase $\phi_{x}(t)$ of the analytic signal are called the canonical amplitude and phase. The rate of change of the canonical phase defines a unique frequency $\omega_{x}(t) \equiv d \phi_{x} / d t$ which is called the instantaneous frequency of the signal (Boashash, 1992). The remainder of this section deals with a practical method for estimating the canonical amplitude and phase and the instantaneous frequency of an unknown signal.

An important limiting case occurs when amplitude and frequency modulation of the signal are relatively weak, in which case the signal is said to be asymptotic. In order for the signal to be asymptotic, the instantaneous frequency must be large compared with the fractional rate of change of the amplitude, i.e.

$\left|\epsilon_{x}(t)\right| \equiv\left|\frac{1}{\omega_{x}} \frac{d \ln A_{x}}{d t}\right| \ll 1$,

together with similar constraints on higher-order derivatives of the amplitude and frequency (Mallat, 1999). When Eq. (5) is satisfied, we will say that the signal has slow amplitude modulation.

Given a real-valued signal $x(t)$, one wishes to determine the associated amplitude $A_{x}(t)$, phase $\phi_{x}(t)$, and 


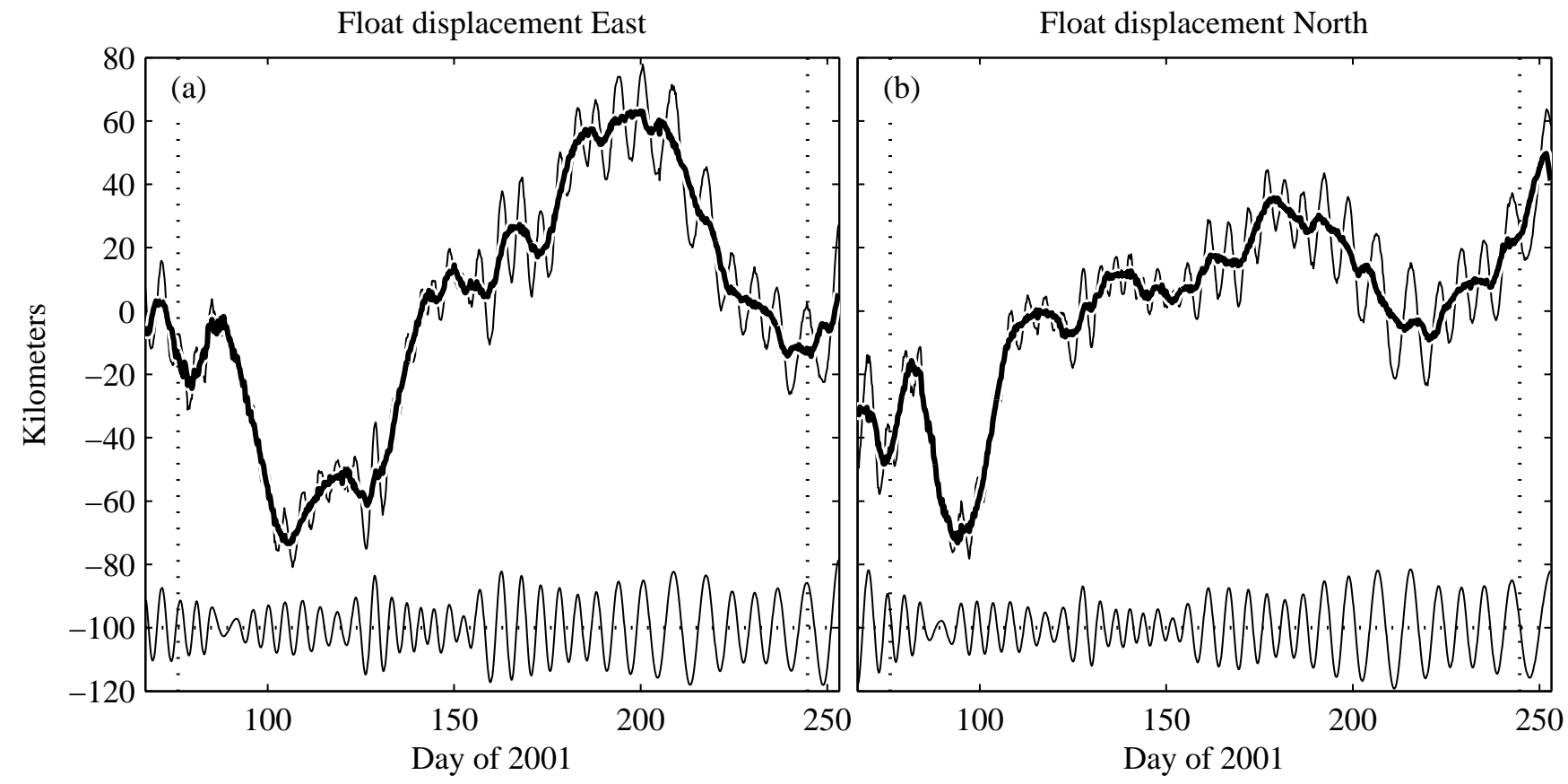

Fig. 2. The eastward (a) and northward (b) locations of the float are shown as the upper thin solid lines. The lower thin solid lines are the diagnosed time-varying elliptical signals, offset by $-100 \mathrm{~km}$, and the thick lines are the residuals. The approximate width of the edge-effect regions are shown with vertical dotted lines.

instantaneous frequency $\omega_{x}(t)$. But generally the signal of interest is embedded in noise or other variability, for example, other amplitude-frequency modulated signals at higher or lower frequencies. For such multi-component or noisy time series, direct construction of the analytic signal, the method described in Appendix A, tends to give meaningless results because it attempts to assign an amplitude and phase to the superposition of all signal components. For this reason, in practical applications the method of wavelet ridge analysis (Delprat et al., 1992; Mallat, 1999) is preferred. This essentially combines a bandpass operation with construction of an analytic signal within each band, forming a redundant decomposition from which the properties of individual amplitude-frequency modulated signals may be determined.

\subsection{The wavelet transform}

The wavelet transform of a signal $x(t)$ with respect to an analytic wavelet $\psi(t)$ is a series of convolutions

$\mathcal{W}_{x}(t, s) \equiv \int \psi_{s}(t-u) x(u) d u \equiv \psi_{s} \star x(t)$

with rescaled versions of the wavelet

$\psi_{s}(t) \equiv \frac{1}{s} \psi\left(\frac{t}{s}\right)$.

The choice of $s$ in the denominator of (7) is one of two common ways of normalizing wavelets across scale, the other being with a $\sqrt{s}$. The "mother" wavelet $\psi(t)$ is zero-mean and has finite energy, and has a Fourier transform

$\Psi(\omega) \equiv \int \psi(t) e^{-i \omega t} d t$

which is here chosen to have a maximum magnitude at $\omega=2 \pi$ with the value of this maximum set to $|\Psi(2 \pi)|=2$. We will use only analytic wavelets, which means $\Psi(\omega)=0$ for $\omega<0$, implying that the time-domain wavelets are complex-valued. It will also be assumed that $\Psi(\omega)$ is real-valued, as is true of the most commonly-used analytic wavelets.

With these choices of normalization, the wavelet transform of a sinusoid $x(t)=\left|A_{o}\right| \cos \left(\omega_{o} t\right)$ is then

$$
\begin{aligned}
\mathcal{W}_{x}(t, s) & =\frac{\left|A_{o}\right|}{2 \pi} \int \Psi(s \omega) e^{i \omega t} \times \\
& \frac{1}{2}\left[2 \pi \delta\left(\omega-\omega_{o}\right)+2 \pi \delta\left(\omega+\omega_{o}\right)\right] d t \\
& =\frac{1}{2}\left|A_{o}\right| e^{i \omega_{o} t} \Psi\left(s \omega_{o}\right)
\end{aligned}
$$

from the convolution theorem and because $\Psi(\omega)$ vanishes for negative frequencies. For our choice of normalization one has $\mathcal{W}_{x}\left(t, 2 \pi / \omega_{o}\right)=\left|A_{o}\right| e^{i \omega_{o} t}$. Thus the scale $s$ has been defined to have the interpretation of a local period, and the wavelet transform magnitude to have interpretation of the local amplitude of oscillations for a real-valued signal. 
The wavelet transform of an amplitude- and frequencymodulated signal $x(t)$ of the form (2) with an analytic wavelet has the approximate form

$\mathcal{W}_{x}(t, s) \approx \frac{1}{2} A_{x}(t) e^{i \phi_{x}(t)} \Psi\left(s \omega_{x}(t)\right)$

a result due to Delprat et al. (1992) and extended by Mallat (1999). The wavelet transform can therefore be used to estimate the canonical amplitude and phase. Specifically, along the scale curve $s(t)=2 \pi / \omega_{x}(t)$ one has

$\mathcal{W}_{x}\left(t, 2 \pi / \omega_{x}(t)\right) \approx A_{x}(t) e^{i \phi_{x}(t)}$

which states that the wavelet transform evaluated along the instantaneous frequency curve is approximately equal to the analytic signal. This shows that in order to estimate the canonical amplitude and phase, we need to first find the instantaneous frequency curve, as is done in the next section.

The approximate equalities in Eqs. (11-12) hold provided that the signal is asymptotic. This means that having slow amplitude variation, condition (5), is necessary but not sufficient to guarantee that the wavelet ridge method will yield accurate estimates of the signal properties.

\subsection{Wavelet ridges}

The method for diagnosing an instantaneous frequency curve from the wavelet transform is called wavelet ridge analysis (Delprat et al., 1992; Mallat, 1999). Writing the wavelet transform as $\mathcal{W}_{x}(t, s)=\left|\mathcal{W}_{x}(t, s)\right| e^{i \Theta_{x}(t, s)}$, one takes the time derivative of the transform phase

$\Omega_{x}(t, s) \equiv \frac{d}{d t} \Theta_{x}(t, s)=\frac{d}{d t} \Im \ln \mathcal{W}_{x}(t, s)$

which will be called the transform frequency; here " $\Im$ " denotes the imaginary part and the " $\Im$ ln" combination has been used to implement the so-called four-quadrant inverse tangent function. For the case of the analyzed signal being an asymptotic signal $x(t)$, the transform frequency is

$$
\begin{aligned}
\Omega_{x}(t, s) & \approx \Im \frac{d}{d t} \ln \left[A_{x}(t) e^{i \phi_{x}(t)}\right]+\Im \frac{\Psi^{\prime}\left(s \omega_{x}(t)\right)}{\Psi\left(s \omega_{x}(t)\right)} s \omega_{x}^{\prime}(t)(14) \\
& \approx \omega_{x}(t)
\end{aligned}
$$

where the second term in Eq. (14) vanishes since $\Psi(\omega)$ has been assumed to be real-valued.

Thus, along the instantaneous frequency curve, the transform frequency is approximately the same as the instantaneous frequency, i.e.

$\Omega_{x}\left(t, 2 \pi / \omega_{x}(t)\right) \approx \omega_{x}(t)$.

This suggests the following method for estimating the unknown instantaneous frequency $\omega_{x}(t)$. Define a curve $\widehat{\omega}_{x}(t)$, called a ridge or ridge curve, satisfying

$\Omega_{x}\left(t, 2 \pi / \widehat{\omega}_{x}(t)\right)=\widehat{\omega}_{x}(t)$ with the individual points along the curve being called ridge points. Such a curve may be diagnosed using a numerical algorithm, as described in Appendix C. It is not guaranteed that a ridge curve exist for all time. Further, in applications, it is usual to consider only ridge points exceeding a certain amplitude, within a specified frequency bound, or contained within a continuous ridge of a least a certain length. Therefore, one often finds ridge curves which break into discrete segments.

Note that if the signal is asymptotic, it follows that $\widehat{\omega}_{x}(t) \approx \omega_{x}(t)$, i.e. the instantaneous frequency curve of the signal is approximately the same as a ridge curve of the wavelet transform. Neglecting the difference between these two curves, we can say that the wavelet ridge algorithm is essentially a diagnosis of the instantaneous frequency curve. More exactly, the wavelet ridge algorithm is a method for estimating the instantaneous frequency curves, and these two sets of curves need not be identical. In the present paper the distinction between ridges and instantaneous frequency curves will not generally be important. After the wavelet ridges have been determined, the canonical amplitude and phase maybe approximately recovered by substituting $\widehat{\omega}_{x}(t)$ for $\omega_{x}(t)$ in Eq. (12).

\section{Time-dependent ellipses}

A complex-valued signal may be represented in several ways: i) in terms of the real-valued component signals; ii) in terms of positively and negatively rotating components; and iii) directly in terms of the physical parameters of the ellipse. In this section we examine the relationships between these different representations, and the means of diagnosing their parameters.

\subsection{Ellipse form}

A natural way to represent an amplitude- and frequencymodulated complex-valued, or bivariate real-valued, time series is in terms of a time-varying ellipse. A fixed ellipse is characterized by an orientation angle $\theta$, a semi-major axis $a$, and a semi-minor axis $b$, as sketched in Fig. 3.

The equation for a time-varying ellipse expressed as a complex-valued time series is

$z(t) \equiv e^{i \theta(t)}[a(t) \cos \phi(t)+i b(t) \sin \phi(t)]$

with $a>|b|>0$. The phase $\phi(t)$ is assumed to be a nondecreasing function of time which expresses the position of a hypothetical "particle" with respect to the major axis of the ellipse. The ellipse is traversed in the counterclockwise (mathematically positive) direction for $b>0$ and in the clockwise direction for $b<0$. The instantaneous radian frequency of the ellipse with respect to phase is $\omega_{\phi}(t) \equiv d \phi / d t>0$, which will be called "orbital frequency", while $\omega_{\theta}(t) \equiv d \theta / d t$ is the rate of precession. Note that $\omega_{\phi}$ is not the same as the angular velocity, discussed later. 


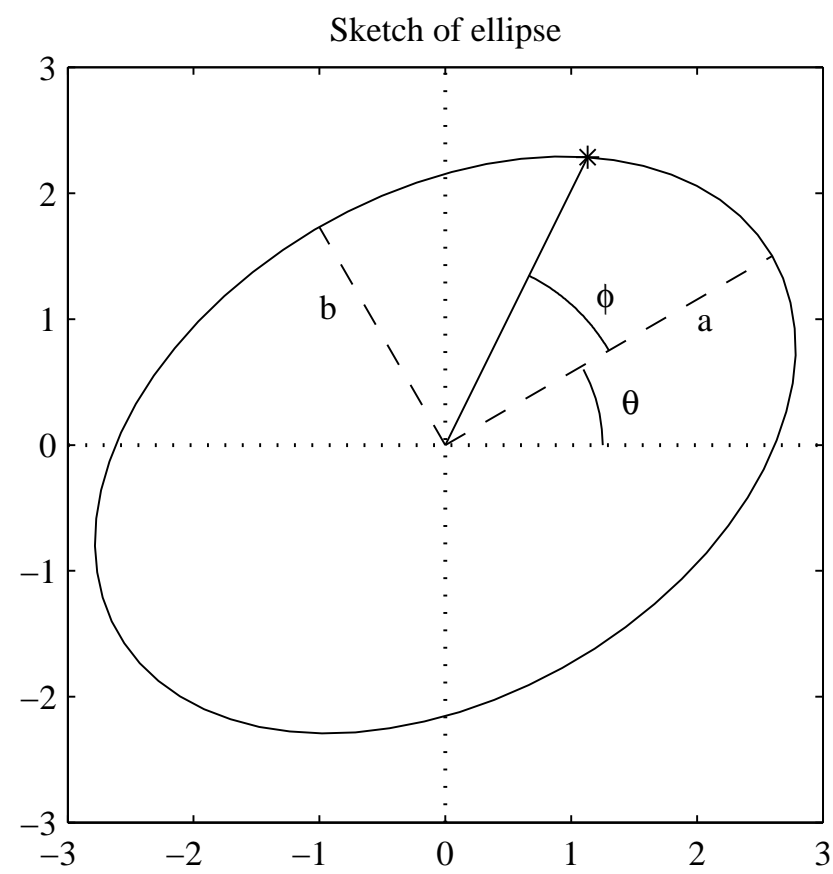

Fig. 3. A sketch of an ellipse with semi-major axis $a=3$ and semiminor axis $b=2$. The orientation of the semi-major with respect to the $\mathrm{x}$-axis is $\theta$, and the instantaneous position of a hypothetical "particle" (marked by an asterisk) with respect to the semi-major axis is $\phi$.

Of all possible rates at which a particle may orbit an ellipse, the parametric form used here (Eq. 18) is special because it describes the particular type of elliptical motion which conserves angular momentum when the orbital frequency $\omega_{\phi}$ and the ellipse geometry are constant. The angular momentum of the (assumed unit mass) particle is

$M(t) \equiv \Re\left\{i z z^{*}\right\}$

[where $z^{\prime}(t) \equiv d z / d t$ and the asterisk denotes the complex conjugate], which, for a constant ellipse geometry $a=a_{o}$, $b=b_{o}, \theta=\theta_{o}$ is

$$
\begin{aligned}
M(t) & =\omega_{\phi}(t) \times \\
& \Re\left\{\left[a_{o} \cos (\phi)+i b_{o} \sin (\phi)\right]\left[-i a_{o} \sin (\phi)+b_{o} \cos (\phi)\right]\right. \\
& =a_{o} b_{o} \omega_{\phi}(t) .
\end{aligned}
$$

It is shown in Appendix B that a fundamental fluid dynamical ellipse, relevant to the data presented earlier, is also orbited in such a way that the "particle" appears to conserve its angular momentum. Thus this signal model is a good match to the physical situation.

\subsection{Cartesian and rotary components}

A complex-valued time series may be written directly as the sum of two real-valued time series

$$
\begin{aligned}
z(t) & =x(t)+i y(t) \\
& =A_{x}(t) \cos \left(\phi_{x}(t)\right)+i A_{y}(t) \cos \left(\phi_{y}(t)\right)
\end{aligned}
$$

where the amplitudes and phases are those of the analytic signals associated with $x(t)$ and $y(t)$, respectively. The realvalued time series $x(t)$ and $y(t)$ will be called the Cartesian components of $z(t)$.

Alternatively $z(t)$ may be expressed as the sum of two counter-rotating circular signals,

$$
\begin{aligned}
z(t) & =z_{+}(t)+z_{-}(t) \\
& =A_{+}(t) e^{i \phi_{+}(t)}+A_{-}(t) e^{-i \phi_{-}(t)}
\end{aligned}
$$

which are called the analytic and anti-analytic components of $z(t)$, constructed as described in Appendix A. The analytic signal $z_{+}(t)$ has a Fourier transform which is supported only on positive frequencies, and hence is an integral over complex exponentials rotating in the mathematically positive (counterclockwise) direction on the complex plane. Similarly the anti-analytic signal $z_{-}(t)$ is an integral over complex exponentials rotating in the mathematically negative (clockwise) direction. Because of this we prefer to call $z_{+}(t)$ and $z_{-}(t)$ the "rotary components" of $z(t)$, by analogy with the use of the term "rotary spectra" in the Fourier analysis of a complex-valued time series (Gonella, 1972).

A subtle point is that $z_{+}(t)$ and $z_{-}(t)$ are not guaranteed to rotate exclusively in the positive and negative directions with time, respectively, although they tend to do so for signals whose spectral energy is concentrated about a central maximum. For such localized signals, our definition of $\phi_{-}(t)$ as the negative of the phase of the anti-analytic signal will then lead to both rotary frequencies $\omega_{+}(t) \equiv d \phi_{+} / d t$ and $\omega_{-}(t) \equiv d \phi_{-} / d t$ being positive.

\subsection{Relations between parameters}

The phases of the rotary components are related to the ellipse phase and orientation via

$\phi_{+}(t)=\phi(t)+\theta(t)$
$\phi_{-}(t)=\phi(t)-\theta(t)$

with the amplitudes of the positively- and negatively-rotating circles related to the semi-major and semi-minor axes through

$$
\begin{aligned}
& A_{+}(t)=[a(t)+b(t)] / 2 \\
& A_{-}(t)=[a(t)-b(t)] / 2
\end{aligned}
$$

so that $A_{+}>A_{-}>0$ for positive $b$ and $A_{-}>A_{+}>0$ for negative $b$. Conversely, the above expressions may be rearranged to give the four ellipse parameters in terms of the four rotary 
parameters $A_{+}, A_{-}, \phi_{+}$, and $\phi_{-}$. Note that $\omega_{+}(t)>0$ and $\omega_{-}(t)>0$ implies $\omega_{\phi}(t)>\left|\omega_{\theta}(t)\right|$ and vice-versa.

One may also find relations between the four Cartesian parameters and the four rotary parameters, by applying the analytic and anti-analytic filters defined in Appendix A to Eqs. (21) and (23). The parameters of the Cartesian components are determined in terms of $A_{+}, A_{-}, \theta$, and $\phi$ as

$$
\begin{aligned}
& \phi_{x}(t)=\phi+\Im \ln \left[A_{+} e^{i \theta}+A_{-} e^{-i \theta}\right] \\
& \phi_{y}(t)=\phi+\Im \ln \left[A_{+} e^{i \theta}-A_{-} e^{-i \theta}\right]-\pi / 2 \\
& A_{x}(t)=\sqrt{A_{+}^{2}+A_{-}^{2}+2 A_{+} A_{-} \cos 2 \theta} \\
& A_{y}(t)=\sqrt{A_{+}^{2}+A_{-}^{2}-2 A_{+} A_{-} \cos 2 \theta}
\end{aligned}
$$

while conversely the rotary parameters are determined from the Cartesian parameters as

$$
\begin{aligned}
\phi_{+}(t) & =\phi_{a}+\Im \ln \left[A_{x} e^{i \phi_{d}}+A_{y} e^{-i \phi_{d}}\right] \\
\phi_{-}(t) & =\phi_{a}+\Im \ln \left[A_{x} e^{i \phi_{d}}-A_{y} e^{-i \phi_{d}}\right] \\
2 A_{+}(t) & =\sqrt{A_{x}^{2}+A_{y}^{2}+2 A_{x} A_{y} \cos 2 \phi_{d}} \\
2 A_{-}(t) & =\sqrt{A_{x}^{2}+A_{y}^{2}-2 A_{x} A_{y} \cos 2 \phi_{d}}
\end{aligned}
$$

where we have defined an average phase and a difference phase as $\phi_{a} \equiv\left[\phi_{x}+\phi_{y}+\pi / 2\right] / 2$ and $\phi_{d} \equiv\left[\phi_{x}-\phi_{y}-\pi / 2\right] / 2$. Comparing these two sets of equations reveals a symmetry between the rotary and the Cartesian formulations.

There therefore exist three sensible ways of describing a time-varying elliptical signal. Each of these three forms is associated with two time-varying amplitudes and two time-varying phases, and therefore also with two frequency curves. Given any one of the three forms, the parameters of the other two may be determined. Note that the six frequencies are all different in general. However, when the ellipse geometry is constant, one has the special case $\omega_{\phi}=\omega_{x}=\omega_{y}=\omega_{+}=\omega_{-}$.

\subsection{Diagnosis with wavelet ridges}

The amplitudes and phases of the Cartesian components may be approximately recovered by applying the wavelet ridge analysis described in Sect. 3.3 to the two Cartesian wavelet transforms $\mathcal{W}_{x}(t, s)$ and $\mathcal{W}_{y}(t, s)$, assuming that the respective signals $x(t)$ and $y(t)$ are asymptotic.

To recover amplitude and phases of the rotary signal components, we define a pair of rotary transforms

$$
\begin{aligned}
& \sqrt{2} \mathcal{W}_{+}(t, s) \equiv \psi_{s} \star z(t)=\sqrt{2}\left[\mathcal{W}_{x}(t, s)+i \mathcal{W}_{y}(t, s)\right] \\
& \sqrt{2} \mathcal{W}_{-}(t, s) \equiv \psi_{s}^{*} \star z(t)=\sqrt{2}\left[\mathcal{W}_{x}^{*}(t, s)+i \mathcal{W}_{y}^{*}(t, s)\right]
\end{aligned}
$$

which have been defined such that $\left|\mathcal{W}_{x}\right|^{2}+\left|\mathcal{W}_{y}\right|^{2}=\left|\mathcal{W}_{+}\right|^{2}+\left|\mathcal{W}_{-}\right|^{2}$. These simplify to

$$
\begin{aligned}
& \sqrt{2} \mathcal{W}_{+}(t, s)=\psi_{s} \star z_{+}(t)+\psi_{s} \star z_{-}(t)=\psi_{s} \star z_{+}(t) \\
& \sqrt{2} \mathcal{W}_{-}(t, s)=\psi_{s}^{*} \star z_{+}(t)+\psi_{s}^{*} \star z_{-}(t)=\psi_{s}^{*} \star z_{-}(t)
\end{aligned}
$$

owing to the analycity of the wavelet. Along the respective instantaneous frequency curves, these become, using the results of Sect. 3.3,

$\mathcal{W}_{+}\left(t, 2 \pi / \omega_{+}(t)\right) \approx \sqrt{2} A_{+}(t) e^{i \phi_{+}(t)}$
$\mathcal{W}_{-}\left(t, 2 \pi / \omega_{-}(t)\right) \approx \sqrt{2} A_{-}(t) e^{-i \phi_{-}(t)}$

again assuming the respective signals are asymptotic.

Wavelet ridge analysis may thus be used to determine the properties of the Cartesian signal components from the Cartesian transforms, or the properties of the rotary signal components from the rotary transforms. All other parameters may be determined from either set of ridges by using the transformation equations of the previous subsection. It is not equivalent to diagnose the ellipse properties using the ridges of the Cartesian transforms versus those using those of the rotary transforms; one or the other set may be more closely asymptotic and therefore better suited to the wavelet ridge analysis. The transformation equations may thus be used to assign a unique pair of rotary components, not themselves asymptotic, by inferring their properties from an asymptotic pair of Cartesian components, and vice-versa. This is illustrated by the example of the next section.

\subsection{Application to the eddy-trapped float}

As an example, we apply the wavelet ridge algorithm to the float data presented in Sect. 2; details of numerical routines used may be found in Appendix C. For wavelets, we prefer the lowest-order generalized Morse wavelets of Olhede and Walden (2002) rather than the more commonly used Morlet wavelets. The generalized Morse wavelets are exactly analytic, which leads to superior performance of the ridgedetection algorithm, particularly for highly time-localized parameter settings. The parameter settings $\beta=4$ and $\gamma=2$, as defined in Olhede and Walden (2002), lead to time-localized wavelets appropriate for this rapidly fluctuating signal. Fifty logarithmically-spaced scale levels are chosen, corresponding to periods of ten to one hundred data points.

Using these wavelets, the Cartesian wavelet transforms $\mathcal{W}_{x}(t, s)$ and $\mathcal{W}_{y}(t, s)$ are formed as in Eq. (6), which are then combined via Eqs. (36) and (37) to yield the rotary wavelet transforms $\mathcal{W}_{+}(t, s)$ and $\mathcal{W}_{-}(t, s)$. Our ridge detection algorithm (see Appendix C) then uses the ridge definition (17) to find, within each transform, all ridge points having greater than unit amplitude and included in a continuous ridge with length at least 1.5 times the corresponding period. Precise frequencies and amplitudes are then found by linearly interpolating the transform between discrete scales, as described in Appendix C.

This process results in a set of four ridge curves $\widehat{\omega}_{x}(t)$, $\widehat{\omega}_{y}(t), \widehat{\omega}_{+}(t)$, and $\widehat{\omega}_{-}(t)$. These are estimates of the underlying instantaneous frequency curves, but are not expected to be identical with them because of contamination by noise and by distortion associated with extraction algorithm. The diagnosed Cartesian and rotary ridge curves are shown in Figs. 4a 
Diagnosed frequencies

Inferred frequencies
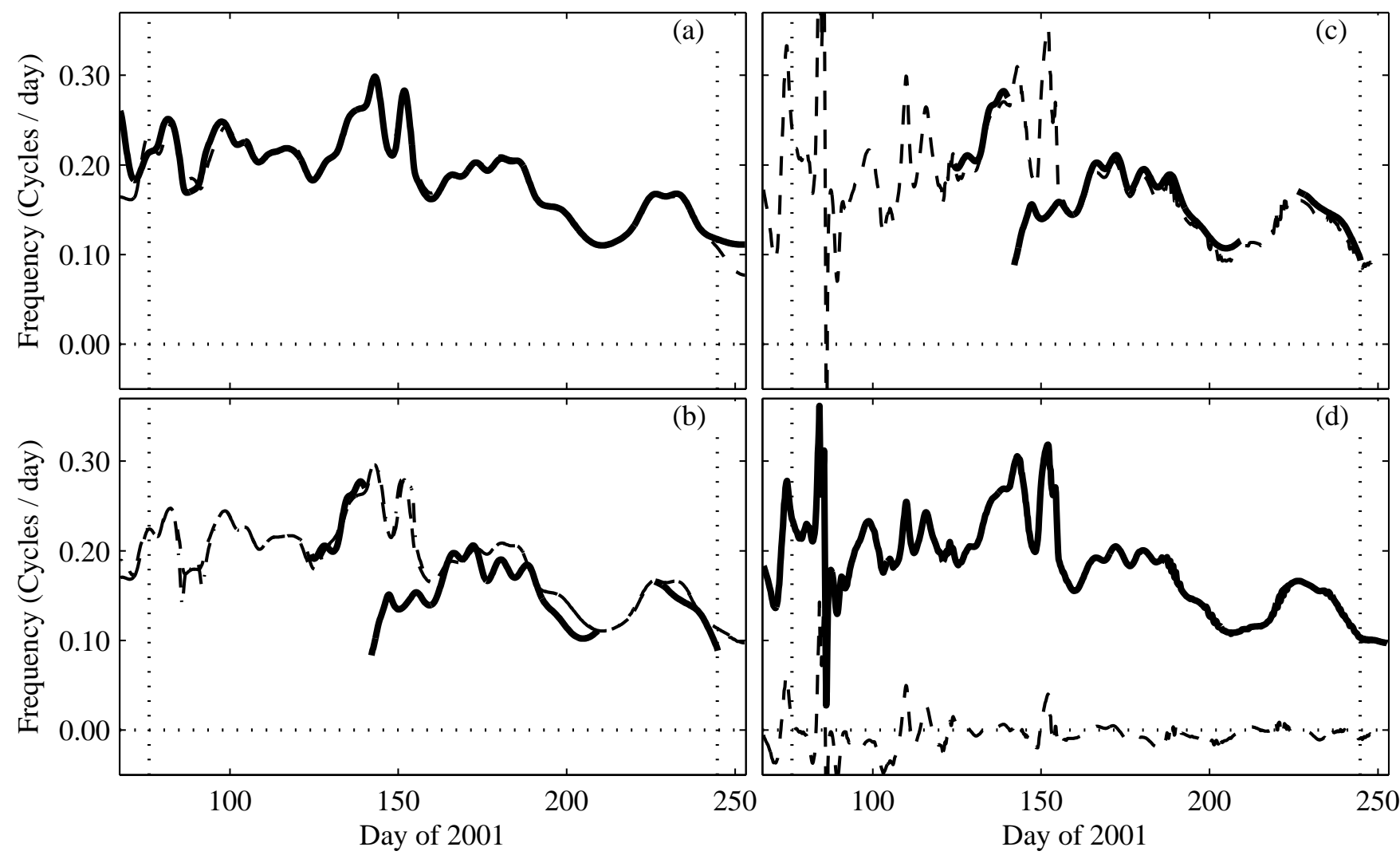

Fig. 4. The frequencies along the Cartesian (a) and rotary (b) ridges, determined as specified in the text. The $x$-component ridges in (a) and positive rotary ridges in (b) are shown as bold lines, while the $y$-component ridges in (a) and negative rotary ridges in (b) are shown as thin dashed lines. A dot-dashed line is shown is also shown in $b$, which is virtually identical to the dotted line; this is the positive rotary frequency curve inferred by the properties along the Cartesian curves. Panel (c) shows the diagnosed (solid line) and inferred (thin dashed line) positive rotary frequency, offset slightly for clarity. Panel (d) shows the orbital frequency $\omega_{\phi}(t)$ and precession rate $\omega_{\theta}(t)$ inferred from the properties along the Cartesian ridges as bold and thin lines, respectively. The approximate width of the edge-effect regions are shown with vertical dotted lines.

and $b$, respectively. For the Cartesian and negative rotary signals, the ridge criteria specified above lead to a set of three virtually identical curves which correspond to the instantaneous frequency curves of the dominant signal, and to none others. The positive rotary transform, however, yields several short ridges which form a broken and incomplete curve. Since the signal rotates in a clockwise sense, the positive rotary transform has much smaller amplitude than the others, and is therefore the most susceptible to noise. Changing parameter settings does not substantially change this performance.

The transformation equations of Sect. 4 may be used to infer the rotary instantaneous frequency curves from the estimated Cartesian instantaneous frequency curves. To do this, estimates of the Cartesian amplitudes and phases - denoted $\widehat{A}_{x}(t), \widehat{A}_{x}(t), \widehat{\phi}_{x}(t)$, and $\widehat{\phi}_{y}(t)-$ are formed by evaluating each Cartesian wavelet transform along its estimated instan- taneous frequency curve, as in Eq. (12). Inserting these amplitude and phase estimates into Eqs. (32-33) and differentiating leads to a second set of estimated rotary frequency curves, which we will say are "inferred" to distinguish them from those "diagnosed" directly by the ridge algorithm.

In fact, Fig. $4 \mathrm{~b}$ already shows the inferred negative rotary curve, which is virtually identical to the diagnosed curve. The diagnosed and inferred positive rotary curves are shown in Fig. 4c. When the diagnosed curve exists, it is virtually identical to the inferred curve, apart from a time in the vicinity of day 150 . At other times the inferred positive rotary curve exhibits large and rapid fluctuations in frequency. This is the reason why it cannot be directly diagnosed: the wavelet ridge algorithm requires a slowly-varying instantaneous frequency curve, and that condition is clearly violated. Closer inspection (not shown) suggests that the period of disagreement in the vicinity of day 150 is a consequence of the 
simultaneous existence of two separate structures in the negative rotary transform.

The inferred orbital frequency $\omega_{\phi}(t)$ and precession rate $\omega_{\theta}(t)$ are shown in Fig. 4d. Like the negative rotary frequency, the orbital frequency exhibits rapid fluctuations during parts of the record. The precession rate is generally very small, apart from a few isolated bursts, but shows a discernible tendency to be negative. On average, the ellipse orientation is drifting slowly in the prograde sense, i.e. in same direction in which the ellipse is being traversed.

After estimating the Cartesian signal properties from the Cartesian ridges, all rotary properties are found from Eqs. (32-35), and then the ellipse properties $a(t), b(t), \phi(t)$, and $\theta(t)$ are found using Eqs. (24-27). A time-varying elliptical signal $z_{e}(t)$ having these properties is constructed via Eq. (18), leading also to a residual $z_{r}(t) \equiv z(t)-z_{e}(t)$. These three signals $z(t), z_{e}(t)$, and $z_{r}(t)$ were presented earlier in Fig. 2. The time-varying elliptical properties $a(t), b(t)$, and $\theta(t)$ are then sampled at a particular time $t_{o}$, and one creates an ellipse by advancing the phase $\phi(t)$ by $2 \pi$ with the ellipse geometry held constant; the ellipse center is considered to be the point $z_{r}\left(t_{o}\right)$. Sampling the time-varying ellipse properties every four days in this way leads to the ellipses shown in Fig. 1.

For this data, an accurate diagnosis of the time-varying elliptical signal was possible from the Cartesian transforms but not the rotary transforms. Yet, the negative rotary instantaneous frequency curve $\omega_{-}(t)$ appears to control the Cartesian instantaneous frequencies. The reasons for these behaviors will become apparent after the perturbation analysis of the next section.

In order for the wavelet ridge analysis to approximately recover the amplitude and phase of the $x$-component signal, it is necessary that the magnitude of $\epsilon_{x}(t)$ defined in Eq. (5) be small, which we say means that $x$ has slowly-varying amplitude. Similar parameters $\epsilon_{-}(t), \epsilon_{+}(t), \epsilon_{-}(t)$ must likewise be small in order for the wavelet ridge analysis to approximately recover the respective signal properties. These four conditions are not identical. It is natural to ask what conditions on the ellipse parameters are required in order that each component signal have slowly-varying amplitude.

\section{The near-circular limit}

The relationships between the elliptic, rotary, and Cartesian forms for a time-varying ellipse may be better seen through creating simplified transformation equations using a small parameter expansion. It will then be apparent that the properties of a time-varying ellipse are often best diagnosed using the Cartesian wavelet transforms.

\subsection{A stronger measure of eccentricity}

The departure of an ellipse from a pure circle is characterized by the eccentricity

$\epsilon(t) \equiv \operatorname{sgn}(b) \sqrt{1-\frac{b^{2}}{a^{2}}}$

which vanishes for purely circular motion and has unit magnitude for purely linear motion. Our definition differs from convention in that negative eccentricities are taken to indicate a negatively-rotating ellipse.

A stronger measure of the departure of an ellipse from a circle will be needed. Such a measure is the "ellipse parameter" (Ruddick, 1987)

$\lambda(t) \equiv \operatorname{sgn}(b) \frac{a^{2}-b^{2}}{a^{2}+b^{2}}=\operatorname{sgn}(\epsilon) \frac{\epsilon^{2}}{2-\epsilon^{2}}=\frac{2 A_{+} A_{-}}{A_{+}^{2}+A_{-}^{2}}$

which has the same limits as the eccentricity, but has $|\lambda|<<|\epsilon|$ for small $|\epsilon|$. For example, the orbit of Pluto, the most eccentric of planetary orbits, has $\epsilon=0.249$ with $\lambda=0.032$, while eccentricity of $\epsilon=0.5$ corresponds to $\lambda=0.143$. To examine the near-circular case, we will perform series expansions in powers of $\lambda$, resulting in simplified approximate expressions valid for small or even moderate eccentricities. Also, we define a measure of the amplitude of the ellipse as

$\kappa(t) \equiv \sqrt{\frac{a^{2}+b^{2}}{2}}=\sqrt{\frac{A_{x}^{2}+A_{y}^{2}}{2}}=\sqrt{A_{+}^{2}+A_{-}^{2}}$

so that $\kappa$ and $\lambda$ contain equivalent information to $a$ and $b$. Expressions for the all amplitude and phase parameters as power series expansions in terms of $\lambda$ are given in Appendix D.

\subsection{Cartesian signals in the near-circular limit}

At this point it is useful to introduce a new notation for the rotary components. For small to moderate eccentricities, the magnitude of one of the two rotary components will be much larger than that of the other. We call the rotary component having the larger amplitude, with properties denoted by the subscript " $>$ ", the "primary" rotary component, and the other the "secondary" rotary component with properties denoted by the subscript " $<$ ". Thus the primary and secondary phases are

$\phi_{>}(t) \equiv \begin{cases}\phi_{+}(t) & A_{+}(t)>A_{-}(t) \\ \phi_{-}(t) & A_{-}(t)>A_{+}(t)\end{cases}$

$\phi_{<}(t) \equiv \begin{cases}\phi_{-}(t) & A_{+}(t)>A_{-}(t) \\ \phi_{+}(t) & A_{-}(t)>A_{+}(t)\end{cases}$

with corresponding frequencies

$\omega_{>}(t) \equiv \frac{d}{d t} \phi_{>}=\omega_{\phi}+\operatorname{sgn}(\lambda) \omega_{\theta}$
$\omega_{<}(t) \equiv \frac{d}{d t} \phi_{<}=\omega_{\phi}-\operatorname{sgn}(\lambda) \omega_{\theta}$ 
and similarly for the amplitudes.

Via the perturbation expansion carried out in Appendix D, the Cartesian frequencies are found to be

$\omega_{x}(t)=\omega_{>}-\frac{1}{2} \frac{d \lambda}{d t} \sin (2 \theta)-\lambda \cos (2 \theta) \omega_{\theta}+\frac{d}{d t} O\left(\lambda^{2}\right)$

$\omega_{y}(t)=\omega_{>}+\frac{1}{2} \frac{d \lambda}{d t} \sin (2 \theta)+\lambda \cos (2 \theta) \omega_{\theta}+\frac{d}{d t} O\left(\lambda^{2}\right)$

which shows that the leading term of both $\omega_{x}(t)$ and $\omega_{y}(t)$ is $\omega_{>}(t)$, the frequency of the primary circle. Thus for

$\omega_{>}(t) \gg\left|\frac{d \lambda}{d t}\right|, \quad \omega_{>}(t) \gg\left|\lambda \omega_{\theta}\right|$

the $x$ - and $y$-frequencies are both approximately the same as the frequency of the primary circle. This verifies that the tendency for the Cartesian instantaneous frequency curves to resemble the primary rotary curve, noted in the discussion of Fig. 4, is in fact a general result.

Similarly one finds the fractional rate of change of the Cartesian amplitudes to be

$$
\begin{aligned}
& \frac{d \ln A_{x}}{d t}=\frac{d \ln \kappa}{d t}+\cos (2 \theta) \frac{1}{2} \frac{d|\lambda|}{d t} \\
&-|\lambda| \sin (2 \theta) \omega_{\theta}+\frac{d}{d t} O\left(\lambda^{2}\right) \\
& \frac{d \ln A_{y}}{d t}=\frac{d \ln \kappa}{d t}-\cos (2 \theta) \frac{1}{2} \frac{d|\lambda|}{d t} \\
&+|\lambda| \sin (2 \theta) \omega_{\theta}+\frac{d}{d t} O\left(\lambda^{2}\right)
\end{aligned}
$$

so that a condition on the rate of change of $\kappa$

$\omega_{>}(t) \gg\left|\frac{d \ln \kappa}{d t}\right|$

is sufficient, together with the two assumptions (51), to imply small $\left|\epsilon_{x}(t)\right|$ and $\left|\epsilon_{y}(t)\right|$. It is therefore the frequency of the primary rotary component which controls the extent to which the Cartesian signals have slowly varying amplitude.

An important corrolary is that there is a distinct difference between prograde precession, $\omega_{\theta}(t) \operatorname{sgn}(\lambda)>0$, and retrograde precession. The above conditions become stronger with retrograde rotation, since $\omega_{>}(t)$ decreases for fixed orbital frequency $\omega_{\phi}(t)$, and weaker with prograde rotation. When the precession rate $\omega_{\theta}(t)$ is of large magnitude relative to the orbital frequency (but still $\left|\omega_{\theta}(t)\right|<\omega_{\phi}$ by assumption), retrograde rotation requires much stronger constraints on the variability of the ellipse geometry in order that the Cartesian signals be asymptotic. This situation is discussed further in the next subsection.

\subsection{Rotary signals in the near-circular limit}

One finds the relative rates of change of the amplitudes of the primary and secondary rotary components are, respectively

$$
\begin{aligned}
& \frac{d}{d t} \ln A_{>}(t)=\frac{d \ln \kappa}{d t}-\frac{\lambda}{4} \frac{d \lambda}{d t}+\frac{d}{d t} O\left(\lambda^{4}\right) \\
& \frac{d}{d t} \ln A_{<}(t)=\frac{d \ln \kappa}{d t}+\frac{d \ln |\lambda|}{d t}+\frac{d}{d t} O\left(\lambda^{3}\right)
\end{aligned}
$$

therefore the condition

$\omega_{>}(t) \gg\left|\lambda \frac{d \lambda}{d t}\right|$

along with Eq. (54) is sufficient to guarantee a slowly varying amplitude of the primary rotary signal, i.e. small $\left|\epsilon_{>}(t)\right|$. On the other hand,

$\omega_{<}(t) \gg\left|\frac{d \ln |\lambda|}{d t}\right|$

is sufficient, together with Eq. (54), to guarantee that the secondary rotary signal has slowly varying amplitude.

When the precession rate $\omega_{\theta}$ is small, (58) is a much stronger constraint on variations of $\lambda$ than is Eq. (57), while the constraint Eq. (51) for the Cartesian signals falls in between. Thus, the conditions that the primary rotary signal, Cartesian signals, and secondary rotary signal be respectively asymptotic imply three increasingly strong constraints on variations of the eccentricity. Variations of the eccentricity with time will cause the ridge algorithm to fail to diagnose the instantaneous frequency curves, and this will happen first for the secondary rotary frequency curve. Thus in order to diagnose the properties of a time-varying, roughly circular and slowly precessing ellipse, one should one should identify $x y$ ridges and then infer the ellipse properties from these, rather than identifying the rotary ridges directly.

When the precession rate is not small, the asymmetry between prograde and retrograde precession appears in the rotary components as well. Other conditions equal, a stronger retrograde rotation tends to bring the primary rotary signal and the Cartesian signals away from the state of being asymptotic, but brings the secondary rotary component towards this state. The primary rotary frequency $\omega_{>}(t)=\omega_{\phi}+\operatorname{sgn}(\lambda) \omega_{\theta}$ can become very small if, as a particle moves around the ellipse, the ellipse also rotates in the opposite direction. In this case, the orbital motion (with respect to the ellipse) in one direction, and the rotation of the ellipse in the other direction, nearly counteract, and the particle is essentially "running in place". While such conditions perhaps lie outside the boundaries for which this model is physically meaningful, it is important to point out that there may be situations in which the rotary components provide the more favorable approach to diagnosing the frequency curves.

\section{Instantaneous and average properties}

Here measures of the ellipse radius and velocity are introduced. In addition to instantaneous measures, which include the rapid variability as the particle orbits the ellipse, one may also form slowly-varying measures which vary only as the ellipse geometry varies. 


\subsection{Radius}

The instantaneous radial distance from the particle to the ellipse center, or "instantaneous radius", is

$R(t) \equiv|z(t)|=\kappa \sqrt{1+|\lambda| \cos (2 \phi)}$

in terms of $\kappa(t), \lambda(t)$, and $\phi(t)$. However, the instantaneous radius varies rapidly due to circulation of the particle around the ellipse, even if the ellipse geometry is fixed. It is desirable to define an average measure which can decouple this rapid oscillation from longer-term changes which reflect variations of the ellipse geometry itself.

One may regard a quantity such as the radius $R(t)$ as a joint function of the ellipse phase $\phi(t)$ and of time with fixed phase, i.e. $R=R(t, \phi(t))$. Then a period-averaged version of $R(t)$

$\bar{R}(t) \equiv \frac{1}{2 \pi} \int_{\phi(t)-\pi}^{\phi(t)+\pi} R\left(t, \phi^{\prime}\right) d \phi^{\prime}$

is formed at every time $t$ by freezing the ellipse properties $a, b$, and $\theta$ and averaging over one period of $\phi$, that is, over one cycle through a fixed ellipse. The period-averaged radius may be determined through the use of an elliptic integral to be

$$
\begin{aligned}
\bar{R}(t)=\frac{2 a}{\pi} E(|\epsilon|) & =\frac{2 \kappa}{\pi} \sqrt{1+|\lambda|} E(|\epsilon|) \\
& =\kappa\left[1-\lambda^{2} / 16+O\left(\lambda^{4}\right)\right]
\end{aligned}
$$

where $E(r)$ is the complete elliptic integral of the second kind as a function of the modulus $r$, with the eccentricity magnitude playing the role of the modulus.

A second measure of the slowly-varying radius is the geometric mean radius

$$
R_{\mathrm{M}}(t) \equiv \sqrt{a|b|}=\kappa \sqrt{\sqrt{1-\lambda^{2}}}=\kappa\left[1-\lambda^{2} / 4+O\left(\lambda^{4}\right)\right]
$$

which determines the area of the ellipse as $\pi R_{\mathrm{M}}^{2}$. While these two measures of the slowly-varying radius are close together in value for small to moderate eccentricities, we prefer the geometric mean radius because of its relationship to area and also its connection with angular momentum discussed shortly.

\subsection{Velocity}

Two rapidly-varying measures of the ellipse velocity are the instantaneous speed $V(t)$ and the instantaneous azimuthal velocity $V_{\Phi}(t)$. The instantaneous speed

$$
V(t) \equiv\left|\frac{d z}{d t}\right|
$$

becomes for constant ellipse geometry (i.e. only the phase $\phi(t)$ varying)

$V(t)=\omega_{\phi}(t) \kappa_{o} \sqrt{1-\left|\lambda_{o}\right| \cos (2 \phi(t))}$ with a period-averaged value $\bar{V}(t)=\omega_{\phi}(t) \bar{R}$. The instantaneous azimuthal velocity $V_{\Phi}(t)$ is found by writing the original time-varying ellipse as

$z(t)=R(t) e^{i \Phi(t)}$

which determines the angular velocity $\omega_{\Phi}(t) \equiv d \Phi / d t$ and hence the azimuth velocity $V_{\Phi}(t) \equiv \omega_{\Phi} R$. Note that angular velocity $\omega_{\Phi}(t)$, the rate of change of azimuthal angle $\Phi(t)$ of the particle, is not the same as the orbital frequency $\omega_{\phi}(t)$, the rate of change of the phase $\phi(t)$ specifying the location of the particle along the ellipse. With constant ellipse geometry, the instantaneous angular velocity is

$\omega_{\Phi}(t) \equiv \frac{d \Phi}{d t}=\operatorname{sgn}\left(\lambda_{o}\right) \frac{R_{M}^{2}}{R^{2}(t)} \omega_{\phi}(t)$

so that the period-averaged azimuthal velocity becomes

$$
\begin{aligned}
\bar{V}_{\Phi}(t) & =\frac{2 b_{o}}{\pi} \omega_{\phi}(t) K\left(\left|\epsilon_{o}\right|\right) \\
& =\operatorname{sgn}\left(\lambda_{o}\right) \kappa_{o} \omega_{\phi}(t)\left[1-5 \lambda_{o}^{2} / 16+O\left(\lambda_{o}^{4}\right)\right]
\end{aligned}
$$

where $K(r)$ is the complete elliptic integral of the first kind with modulus $r$.

However these simple expressions for the speed and azimuthal velocity are only sensible when the ellipse geometry is constant, that is, when variations in ellipse geometry and orientation do not contribute to the velocity. Therefore we will construct slowly-varying measures which are true generally, but which reduce to simple expressions when the ellipse geometry is constant.

To do so we treat the velocity time series $z^{\prime}(t)$ as timevarying elliptical signal in its own right, and determine its time-varying parameters. In fact the parameters of the velocity ellipse, i.e. the ellipse associated with $z^{\prime}(t)$, are determined immediately from those of the "position" ellipse of $z(t)$, as shown in Appendix D. Then forming a period-averaged radius and geometric mean radius as in the preceding subsection, but using the ellipse parameter values of the velocity ellipse, defines a period-averaged speed $\bar{V}(t)$ and geometric mean velocity $V_{M}(t)$. For a constant ellipse geometry the ellipse speed so constructed reduces to Eq. (64), while the geometric mean velocity becomes $V_{M}(t)=\operatorname{sgn}(\lambda) \omega_{\phi}(t) R_{M}$.

We prefer the instantaneous radius $R(t)$ and instantaneous azimuthal velocity $V_{\Phi}(t)$ as rapidly-varying measures of the ellipse properties, and geometric mean radius $R_{M}(t)$ and geometric mean velocity $V_{M}(t)$ as corresponding slowlyvarying measures. Both pairs determine the angular momentum when the ellipse geometry is constant

$M(t)=a_{o} b_{o} \omega_{\phi}(t)=V_{\Phi}(t) R(t)=R_{M}(t) V_{M}(t)$

but the former pair varies rapidly throughout an orbit while the latter pair does not. 


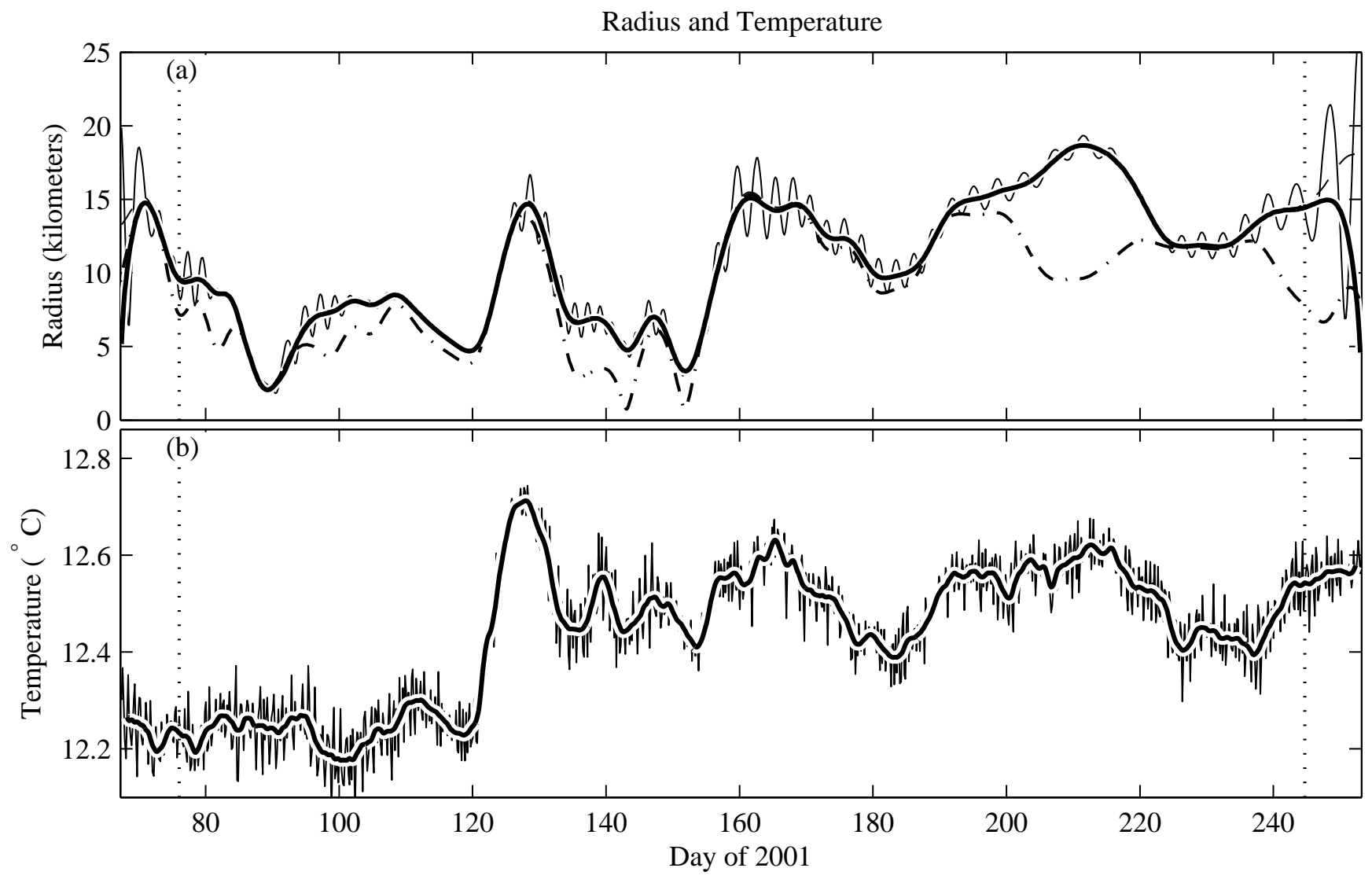

Fig. 5. Panel (a) shows the instantaneous radius $R(t)$ as a thin solid line and the period-averaged radius $\bar{R}(t)$ as a heavy solid line. The dash-dotted line is an estimated radius using a fixed-frequency bandpass filter as described in the text. In panel (b), the in situ temperature is shown as a thin solid line, and heavy solid line is the temperature smoothed with a 12-point Hanning filter. The approximate width of the edge-effect regions are shown with vertical dotted lines.

\subsection{Application to the eddy-trapped float}

The instantaneous and mean radius of the time-varying elliptical signal diagnosed earlier using the Cartesian ridges of the float time series are shown in Fig. 5a. The mean radius is indeed slowly-varying, as desired, while the instantaneous curve exhibits high-frequency fluctuations about the mean curve. It is important to emphasize that no additional explicit filtering has been performed; there is no need, since the ellipse properties extracted from the ridge are have essentially already been smoothed in proportion to the local period by the wavelet analysis.

For comparison, Fig. $5 \mathrm{~b}$ shows the temperature recorded by the float. After day 120, the period-averaged radius and temperature variations are remarkably similar; but note that the high-frequency fluctuations present in the instantaneous radius are not apparent in the temperature. Variations in radius could mean either that the float is observing a change in the eddy structure, or that the float crosses material surfaces to move to a new position inside a fixed eddy. In the lat- ter case one should expect to see changes in the temperature, but not in the former case. The correspondence seen in Fig. 5 is therefore compelling evidence that the float is "profiling" through a fixed eddy - crossing material surfaces - at long time scales but not at the short orbital time scale.

A simpler estimate of radius may also be formed by a fixed-frequency bandpass filter. In fact, the negative rotary transform evaluated at a fixed scale is an example of such a bandpass. The negative rotary transform magnitude (divided by $\sqrt{2}$ ) evaluated at the scale for which the transform has the largest time-mean magnitude is also shown in Fig. 5a. Before day 180 , the agreement with $R(t)$ is often good but also shows periods of substantial underestimation. After day 180, however, the agreement is poor, and no longer matches the temperature fluctuations; the float appears to be profiling the eddy for only half the time suggested by the period-mean radius.

The instantaneous radius versus azimuthal velocity, together with the geometric mean radius and geometric mean velocity, are presented in Fig. 6; the "edge-effect" regions 

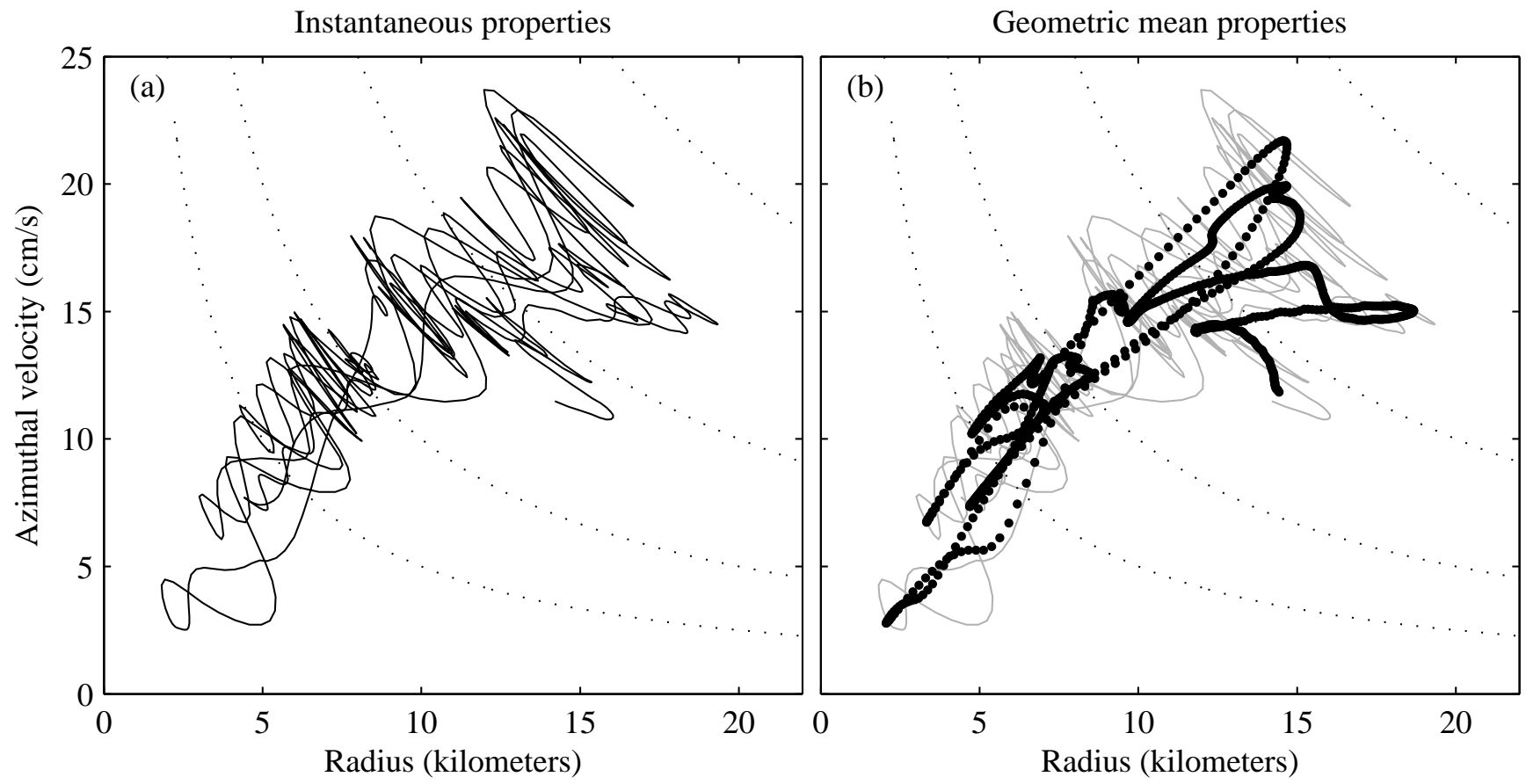

Fig. 6. The instantaneous radius $R(t)$ versus $\left|V_{\Phi}(t)\right|$, the magnitude of the instantaneous azimuthal velocity (a) and the geometric mean radius $R_{M}(t)$ and $\left|V_{M}(t)\right|$, the magnitude of the geometric mean velocity $(\mathbf{b})$. In (b), the instantaneous values are plotted as a light gray line and the average values as a heavy black line. Dotted curves are proportional to $1 / R$.

near either end of the time series have been omitted. It is usual to make such radius-velocity scatter plots when examining oceanic eddies (e.g. Prater and Sanford, 1994), because a circular eddy in solid-body rotation will have speed proportional to radial distance within its central core. Here the instantaneous properties show large scatter, but much of this scatter is oriented along lines such that $V_{\Phi} R$ is constant, that is, lines along which angular momentum is conserved. The corresponding average values collapse onto an almost perfectly straight line. This is precisely the behavior predicted for a circular vortex deformed by strain (Appendix B), and suggests that the scatter of the instantaneous properties is not "noise", as it might appear at first glance, but rather is information regarding the degree of eccentricity of the eddy.

It should be pointed out that the assumed signal form essentially imposes that the high-frequency variations should conserve angular momentum. If the high-frequency fluctuations exhibited some other behavior, it might not be captured by this method. Nevertheless, judging from the smoothly varying residual curve in Fig. 2 even at short time scales, the assumed form appears to be a good match for the variability present in the data, in addition to matching the physical model of a circular eddy deformed by strain.

\section{Discussion}

A method for diagnosing the properties of a time-varying elliptical signal has been presented and applied to a position record from a subsurface oceanographic float. This method has all the advantages of the wavelet ridge analysis on which is based - it is highly flexible, involves very few parameter choices, is reproducible, affords a rigorous statistical treatment, and also generates results which agree with one's intuitive notion of correctness. What is new in this treatment is the ability to interpret the information encoded in the wavelet ridge analysis in terms of geometric quantities, to identify the different pathways by which equivalent information may be found, and to construct average quantities which decouple the fast orbital motion from slower structural changes.

It was seen that there exist three representations of a timevarying elliptical signal which incorporate equivalent information: in terms of the Cartesian components, in terms of the rotary or analytic and anti-analytic components, and directly in terms of time-varying ellipse parameters. Yet, it was shown that under many circumstances, it may be easier to diagnose the signal properties from the ridges of the Cartesian wavelet transforms, and then to infer other properties using the transformation equations derived in Sect. 4.3. But since there are a number of special situations - for example, the difference between prograde and retrograde precession - 
we have provided the tools necessary to assess the parameter space for a given application and then to identify the appropriate procedure. It should also be pointed out that the particular parameters which appeared as the most physically interesting in this application may not be so in other instances. For example, here the negative rotary frequency appears as the essential quantity, but in an analysis of planetary orbits one might expect the orbital frequency and precession rate to be the most relevant.

In the application, it was found that this method could extract the time-varying elliptical signal, leaving behind a residual containing only very minor fluctuations at the ellipse period, despite the fact that the residual itself presents substantial and often rapid variability. A display of the looping time series can then be replaced with a more detailed plot showing the estimated instantaneous geometry of the time-varying elliptical signal. Examination of mean quantities over an orbit support the hypothesis that slow variations in the ellipse structure are due to the float migrating across material surfaces. This is seen in two ways: first, the geometric mean radius - but not the simple bandpass estimate of radius closely follows changes in temperature; second, the geometric mean radius versus geometric mean velocity collapse to a line, suggesting an underlying eddy in near solid-body rotation. While the reason for the elliptical trajectories has not been demonstrated here, a likely candidate is the distortion of a circular eddy into an ellipse by a background strain field.

We believe that this method will be applicable in a broad range of circumstances. However, it is important to address the role of randomness, and in particular of instrument noise. Instrument noise can easily generate nonzero eccentricities, particularly if the noise is anisotropic, which may indeed be the case in the data presented here. Noise may also lead to spurious fluctuations in the instantaneous frequency curves, and is expected to be particularly troublesome when inferring a weaker signal component from two stronger diagnosed components. It is possible to create rigorous statistical estimators, together with confidence intervals, for all the important physical properties, a task which is currently being undertaken.

The present work has focused on establishing the properties of the method. For the specific problem of the eddytrapped float, one may now turn to addressing interesting physical questions. For example, one would like to distinguish between different types of transitions: 1) float profiling within a fixed eddy; 2) conservative adjustment, i.e. eddy evolution conserving mass and circulation; and 3) nonconservative transitions, e.g. eddy mergers or mass expulsions. Building on the work presented here, one may form quantities expected to be conserved under different types of transitions, so that identifying and classifying such transitions may be approached as a statistical problem.

This method represents a new tool for the study of Lagrangian turbulence. It is different from other methods currently used for treating Lagrangian data in that it is grounded in time/frequency theory. This permits the fundamental time dependence of the observed quantities to be explicitly resolved. However, the relationship between this "Lagrangian time/frequency" perspective and the underlying Eulerian structures of basic fluid turbulence (e.g. McWilliams, 1984; Lapeyre et al., 2001) needs to be established. By applying this method to idealized numerical models, one could gain valuable insight into the performance of the method and its interpretation. These lessons could then be applied when studying the real ocean, where it is hoped this method could substantially increase our understanding of the behavior of oceanic coherent eddy structures.

\section{Appendix A}

\section{Construction of the analytic signal}

The analytic signal (Boashash, 1992) is constructed through a time-domain convolution,

$x_{+}(t) \equiv 2 \mathcal{A} \star x(t) \equiv A_{x}(t) e^{i \phi_{x}(t)}$,

of the original signal with the "analytic filter"

$\mathcal{A}(t) \equiv \frac{1}{2}\left[\delta(t)+\frac{i}{t \pi}\right]$.

The Fourier transform of the analytic filter is the unit step function. This filtering operation therefore corresponds in the frequency domain to setting the amplitudes of all negatively-rotating Fourier components to zero, and doubling the amplitudes of all positively-rotating Fourier components. The original signal is recovered by $x(t)=\Re\left\{x_{+}(t)\right\}=\left[x_{+}(t)+x_{+}^{*}(t)\right] / 2$.

One may also form an analytic and an anti-analytic signal associated with a complex-valued time series $z(t)$. We define the analytic and anti-analytic signals of a complexvalued time series as

$z_{+}(t) \equiv \mathcal{A} \star z(t) \equiv A_{+}(t) e^{i \phi_{+}(t)}$
$z_{-}(t) \equiv \mathcal{A}^{*} \star z(t) \equiv A_{-}(t) e^{-i \phi_{-}(t)}$

in terms of which the original time series is recovered by $z(t)=z_{+}(t)+z_{-}(t)$. Note the difference in definition of the analytic signal between the real-valued and complex-valued, and also in the reconstruction formula. This is done in order that amplitude have an intuitive interpretation in each case.

\section{Appendix B}

\section{A fluid dynamical ellipse}

In this section it is shown that the ellipse traced out by a fluid parcel orbiting an elliptical streamfunction conserves the angular momentum of the parcel. A streamfunction $\Upsilon(x, y)$ defines the horizontal velocity vector through $\boldsymbol{u}=\hat{\boldsymbol{k}} \times \nabla \Upsilon$ where 
$\hat{\boldsymbol{k}}$ is the vertical unit vector, " $x$ " denotes the vector cross product, and $\nabla$ is the horizontal gradient operator. There is therefore no flow across streamlines, i.e. contours of constant stream function, because $\boldsymbol{u} \cdot \nabla \Upsilon=0$. The elliptical streamfunction

$\Upsilon(x, y)=\frac{x^{2}}{a^{2}}+\frac{y^{2}}{b^{2}}$

is constant along ellipses with semi-major axes $c a$ and semiminor axes $c b$, for any constant $c$.

Let the azimuthal and radial coordinates be $\Phi$ and $R$; here, these are independent variables, as opposed to parametric functions of time as in the rest of the paper. In polar coordinates, the streamfunction is

$\Upsilon(R, \Phi)=R^{2}\left[\frac{\cos ^{2} \Phi}{a^{2}}+\frac{\sin ^{2} \Phi}{b^{2}}\right]$

with azimuthal velocity

$V_{\Phi}(R, \Phi)=\frac{\partial \Upsilon}{\partial R}=\frac{2}{R} \Upsilon(R, \Phi)$.

Thus, the angular momentum of a unit-mass parcel $M=V_{\Phi} R$ is constant along a streamline. Alternatively, we may simply note that between two elliptical streamlines, $V_{\Phi} \Delta R$ must be constant by continuity, but $\Delta R$ is proportional to $R$ and hence $V_{\Phi} R$ must also be constant.

The ellipse traced out by a fluid particle orbiting an elliptical streamfunction is therefore a constant-frequency ellipse, shown in Sect. 4.1 to conserve angular momentum. Such a streamfunction is created by a constant vorticity superimposed on a constant strain,

$\Upsilon(x, y)=\frac{Q}{4}\left[x^{2}+y^{2}\right]+\frac{S}{4}\left[x^{2}-y^{2}\right]$

where $Q$ is the vertical component of the vorticity and $S$ is the rate of strain, directed at forty-five degrees to the $\mathrm{x}$ axis. For $Q>0$ and $|S|<Q$ this is an elliptical streamfunction with ellipse parameter $\lambda=-S / Q$ and with major axis oriented along the $\mathrm{x}$-axis.

This very simple discussion applies to a purely twodimensional eddy. However, Ruddick (1987) showed that a more realistic model of an oceanic coherent eddy subjected to strain behaves similarly, with an initially circular eddy again distorting to become an ellipse.

\section{Appendix C}

\section{Notes on computations}

All functions necessary to perform the wavelet ridge analysis presented in this paper are included in the Jsignal module of Jlab, a freeware Matlab toolbox available at the first author's web site, http://www.jmlilly.net. All functions were written or co-written from scratch by the first author, and require no additional toolboxes other than those included with standard Matlab. In the interest of presenting high quality-software, heavy use is made of automated testing. At this writing, a new version of Jlab, with new functions, improvements, and bug fixes, is being posted every few months.

Most of the functions pertaining to diagnosing the properties of an elliptical time series are straightforward applications of equations presented here and are fully explained by the documentation. Routine ecconv converts between different eccentricity measures, such as $\lambda$ and $\varepsilon$. The transformation equations of Sect. 4.3 are implemented by ellconv. The properties of the velocity ellipse are determined by elldiff using the equations presented in Appendix D. Measures of the instantaneous and mean radius and velocity, presented in Sect. 6, are implemented by ell rad and ellvel, respectively. The routine ellipseplot is a plotting tool.

The wavelet transform and ridge analysis itself, however, are involved computations, and therefore some additional comments seem appropriate. The generalized Morse wavelets are computed by mor sewave, taking into account the fact that these wavelets are defined in the frequency domain. The wavelet transform, Eq. (6), is implemented by wavetrans. This routine uses a fast frequency-domain algorithm, permits different choices of boundary conditions at the time series endpoints, and may be applied to a multicomplement dataset stored as a matrix.

The ridges - curves satisfying Eq. (17) - are found by ridgewalk using a fast and nearly loopless algorithm to identify the ridge points, which are then chained together into ridge curves. Ridge curves do not wrap around from the end to the beginning of the time series. Occasional ambiguities in chaining ridge points together are resolved by attempting to make the amplitude along the ridge continuous. The user may specify both the minimum amplitude of a ridge point, and the minimum length of a ridge curve. This routine also supports transforms of multi-component datasets. The output ridge parameters may be converted from head-to-tail column vectors to arrays and back using the mat $2 \mathrm{col}$ and col2mat from the Jdata module.

The transform values are interpolated to the exact ridge location, which generally lies between discrete levels of the wavelet transform, using ridgeinterp. This leads to a substantial improvement in the behaviors of frequencies derived from the ridge phases.

\section{Appendix D}

\section{Expansions for small eccentricity}

In terms of $\kappa$ and $\lambda$, the semi-major and semi-minor axes are

$$
\begin{aligned}
a(t) & =\kappa \sqrt{1+|\lambda|} \\
|b(t)| & =\kappa \sqrt{1-|\lambda|}
\end{aligned}
$$


while the Cartesian amplitudes $A_{x}$ and $A_{y}$ [ (30) and (31)] are

$$
\begin{aligned}
& A_{x}(t)=\kappa \sqrt{1+|\lambda| \cos (2 \theta)} \\
& A_{y}(t)=\kappa \sqrt{1-|\lambda| \cos (2 \theta)}
\end{aligned}
$$

and finally the primary and secondary rotary amplitudes are

$$
\begin{aligned}
& A_{>}(t)=\frac{\kappa}{\sqrt{2}} \sqrt{1+\sqrt{1-\lambda^{2}}} \\
& A_{<}(t)=\frac{\kappa}{\sqrt{2}} \sqrt{1-\sqrt{1-\lambda^{2}}} .
\end{aligned}
$$

The phase of the $x$-component, momentarily expressed in terms of the amplitudes of the primary and secondary rotary components, is

$$
\begin{aligned}
\phi_{x}(t)=\phi+\Im \ln [ & \left.A_{>} e^{i \operatorname{sgn}(\lambda) \theta}+A_{<} e^{-i \operatorname{sgn}(\lambda) \theta}\right] \\
=\phi+\operatorname{sgn}(\lambda) \theta & \\
& \quad+\Im \ln \left[1+\left(A_{<} / A_{>}\right) e^{-i 2 \operatorname{sgn}(\lambda) \theta}\right]
\end{aligned}
$$

while the phase of the $y$-component is

$$
\begin{aligned}
& \phi_{y}(t)=\phi+\Im \ln \left[\operatorname{sgn}(\lambda) A_{>} e^{i \operatorname{sgn}(\lambda) \theta}\right. \\
&\left.-\operatorname{sgn}(\lambda) A_{<} e^{-i \operatorname{sgn}(\lambda) \theta}\right]-\pi / 2 \\
&=\phi+\operatorname{sgn}(\lambda)[\theta-\pi / 2] \\
&+\Im \ln \left[1-\left(A_{<} / A_{>}\right) e^{-i 2 \operatorname{sgn}(\lambda) \theta}\right] .
\end{aligned}
$$

The amplitudes become

$$
\begin{aligned}
& a(t)=\kappa\left[1+|\lambda| / 2-\lambda^{2} / 8+|\lambda|^{3} / 16+O\left(\lambda^{4}\right)\right] \\
&|b(t)|=\kappa\left[1-|\lambda| / 2-\lambda^{2} / 8-|\lambda|^{3} / 16+O\left(\lambda^{4}\right)\right] \\
& A_{x}(t)=\kappa[1+|\lambda| \cos (2 \theta) / 2 \\
&\left.-\lambda^{2} \cos ^{2}(2 \theta) / 8+O\left(|\lambda|^{3}\right)\right] \\
& A_{y}(t)=\kappa[1-|\lambda| \cos (2 \theta) / 2\left.-\lambda^{2} \cos ^{2}(2 \theta) / 8+O\left(|\lambda|^{3}\right)\right] \\
& A_{>}(t)=\kappa\left[1-\lambda^{2} / 8+O\left(\lambda^{4}\right)\right] \\
& 2 A_{<}(t)=\kappa\left[|\lambda|+|\lambda|^{3} / 8+O\left(|\lambda|^{5}\right)\right]
\end{aligned}
$$

when expressed as series expansions with respect to $\lambda$. To expand the Cartesian phases, note

$$
A_{<} / A_{>}=|\lambda| / 2+|\lambda|^{3} / 8+O\left(|\lambda|^{5}\right)
$$

together with

$$
\ln (1+r)=r-r^{2} / 2+O\left(r^{3}\right)
$$

valid for $|r|^{2}<1$ (Dwight, 1961, \# 601). One then finds the phases of the $x$ - and $y$-components are

$$
\begin{aligned}
\phi_{x}(t)=\phi+\operatorname{sgn}(\lambda) \theta & -\lambda \sin (2 \theta) / 2 \\
& +\lambda|\lambda| \sin (4 \theta) / 8+O\left(\lambda^{3}\right) \\
\phi_{y}(t)=\phi+\operatorname{sgn}(\lambda)[\theta & -\pi / 2]+\lambda \sin (2 \theta) / 2 \\
& +\lambda|\lambda| \sin (4 \theta) / 8+O\left(\lambda^{3}\right)
\end{aligned}
$$

from which Eqs. (49) and (50) follow.

\section{Appendix E}

\section{The velocity ellipse}

The parameters of the velocity ellipse are immediately determined from those of the position ellipse. Writing

$z^{\prime}(t)=\widetilde{A}_{+}(t) e^{i \widetilde{\phi}_{+}(t)}+\widetilde{A}_{-}(t) e^{-i \widetilde{\phi}_{-}(t)}$

and noting that $z_{+}^{\prime}(t)$ and $z_{-}^{\prime}(t)$ remain analytic and antianalytic, respectively, one finds

$$
\begin{aligned}
& \widetilde{\phi}_{+}(t)=\phi_{+}(t)+\Im \ln \left(\frac{d \ln A_{+}}{d t}+i \frac{d \phi_{+}}{d t}\right) \\
& \widetilde{\phi}_{-}(t)=\phi_{-}(t)+\Im \ln \left(\frac{d \ln A_{-}}{d t}+i \frac{d \phi_{-}}{d t}\right) \\
& \widetilde{A}_{+}(t)=A_{+}(t) \times \sqrt{\left(\frac{d \phi_{+}}{d t}\right)^{2}+\left(\frac{d \ln A_{+}}{d t}\right)^{2}} \\
& \widetilde{A}_{-}(t)=A_{-}(t) \times \sqrt{\left(\frac{d \phi_{-}}{d t}\right)^{2}+\left(\frac{d \ln A_{-}}{d t}\right)^{2}} .
\end{aligned}
$$

However, determination of the parameters of instantaneous frequency curves of $z(t)$ from those of its derivative $z^{\prime}(t)$ is not so simple, because it would involve solving two sets of two coupled nonlinear differential equations. The periodaverage speed, root-mean-square speed, and geometric mean velocity can now be found by using the comparable measures defined for the radius, substituting the ellipse parameters of the velocity ellipse for those of the position ellipse. In the above equations one notices the occurrence of the " $\epsilon$ " parameters which are the ratios of a fractional rate of change of amplitude to a frequency.

Acknowledgements. The first author was supported by a Chateaubriand Fellowship from the French government, by a fellowship from the Conseil Scientifique of the Université Pierre et Marie Curie in Paris, and by award \#0526297 from the Physical Oceanography program of the National Science Foundation. He wishes to acknowledge the work of F. Rekibi in co-authoring some software routines, the keen observations of L. Bunge which helped to improve early versions of the key algorithms, and many interesting conversations with S. Olhede. Careful readings by two reviewers are appeciated and have led to an improved manuscript.

Edited by: H. A. Dijkstra

Reviewed by: P. J. van Leeuwen and another referee 


\section{References}

Boashash, B.: Estimating and interpreting the instantaneous frequency of a signal - Part 1: Fundamentals, Proc. IEEE, 80, 520538, 1992.

Delprat, N., Escudié, B., Guillemain, P., Kronland-Martinet, R., Tchamitchian, P., and Torrésami, B.: Asymptotic wavelet and Gabor analysis: extraction of instantaneous frequencies, IEEE Trans. Inform. Theory, 38, 644-665, 1992.

Dwight, H. B.: Tables of integrals and other mathematical data, Macmillian Publishing Co., Inc., New York, fourth edition, 1961.

Gonella, J.: A rotary-component method for analyzing meteorological and oceanographic vector time series, Deep-Sea Res., 19, 833-846, 1972.

Lankhorst, M. and Zenk, W.: Lagrangian observations of the middepth and deep velocity fields of the northeastern Atlantic Ocean, J. Phys. Oceanogr., 36, 43-63, 2006.

Lapeyre, G., Hua, B. L., and Klein, P.: Dynamics of the orientation of active and passive scalars in two-dimensional turbulence, Phys. Fluids, 13, 251-264, 2001.
Mallat, S.: A wavelet tour of signal processing, Academic Press, San Diego, 1999.

McWilliams, J. C.: The emergence of isolated coherent vortices in turbulent flow, J. Fluid Mech., 146, 21-43, 1984.

McWilliams, J. C.: Submesoscale coherent vortices in the ocean, Rev. Geophys., 23, 165-182, 1985.

Olhede, S. C. and Walden, A. T.: Generalized Morse wavelets, IEEE Trans. Signal Processing, 50, 2661-2670, 2002.

Prater, M. D. and Sanford, T.: A Meddy off Cape Vincent, I: Description, J. Phys. Oceanogr., 24, 1572-1586, 1994.

Rossby, H., Dorson, D., and Fontain, J.: The RAFOS System, J. Atmos. Ocean Tech., 3, 672-679, 1986.

Ruddick, B. R.: Anticyclonic lenses in large-scale strain and shear, J. Phys. Oceanogr., 17, 741-749, 1987.

Veneziani, M., Griffa, A., Reynolds, A. M., Garraffo, Z. D., and Chassignet, E. P.: Parameterizations of Lagrangian spin statistics and particle dispersion in the presence of coherent vortices, J. Mar. Res., 63, 1057-1083, 2005. 